\title{
Laminate circular cylindrical shell
}

\author{
Eva Kormaníková ${ }^{1, *}$, and Kamila Kotrasová ${ }^{1}$ \\ ${ }^{1}$ TUKE Košice, Civil Engineering Faculty, Vysokoškolská 4, 04200 Košice, Slovakia
}

\begin{abstract}
The paper deals with a numerical approach of optimal design modelling of laminate circular cylindrical shell. For thin-walled shells the classical shell theory is capable of accurately predicting the shell behaviour. In the frame of numerical optimization of circular cylindrical shell there is made the minimization of weight subject to displacement constraint. Thickness of layers is taken as design variable. In the example there is found the thickness of the shell laminate roof under constant pressure loading using Modified Feasible Direction method.
\end{abstract}

\section{Introduction}

For many years, designers have treated optimization problems involving composite materials. The design of a composite laminate stacking sequence generally involves selecting discrete layer thickness - a discrete optimization problem. The directionality of fibre composites needs the optimization in the process of design of these materials. Many works have been done on optimization of cylindrical shells, for example, Park et al. [1] used optimization of laminate stacking sequence to maximize the strength. Adali and Verijenko [2] optimized the stacking sequence design for hybrid laminates. Soremekun et al. [3] used optimization algorithm for stacking sequence blending of multiple composite laminates to minimize the weight and the cost of the panels. Weaver [4] used computational study for designing the laminate composite cylindrical shells under axial compression to minimize the mass with local and global constraints.

\section{Classical shell theory}

Thin-walled laminate shells can be also modelled as twodimensional structural elements but with single or double curved reference surfaces (Fig. 1).

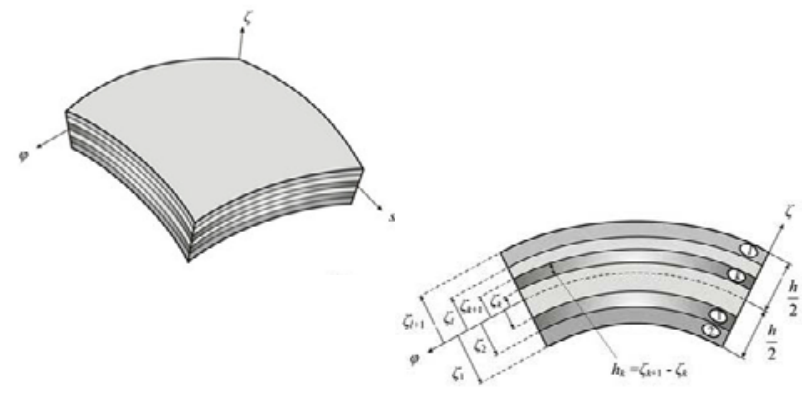

Fig. 1. Double curved laminated shell and layout of layers [5]
The modelling and analysis of laminate circular cylindrical shells fabricated from fibre composite material depends on the radius/thickness ratio (Fig. 2).

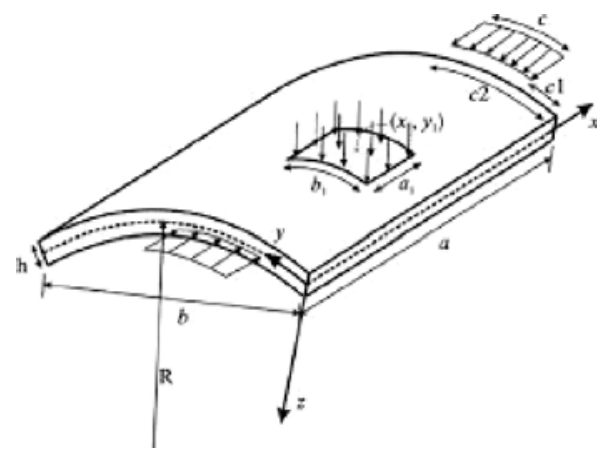

Fig. 2. Circular cylindrical shell under general loading [6]

The strain displacement relations for a circular cylindrical shell using Love's first approximation are given by

$$
\begin{gathered}
\bar{\varepsilon}_{x}=\frac{\partial u}{\partial x} \\
\bar{\varepsilon}_{s}=\frac{\partial v}{\partial s}+\frac{w}{R}, \quad \bar{\varepsilon}_{x s}=\frac{\partial u}{\partial s}+\frac{\partial v}{\partial x}, \\
\kappa_{x}=-\frac{\partial^{2} w}{\partial x^{2}}, \kappa_{s}=-\frac{\partial^{2} w}{\partial s^{2}}+\frac{1}{R} \frac{\partial v}{\partial s}, \\
\kappa_{x s}=-2 \frac{\partial^{2} w}{\partial x \partial s}+\frac{1}{R} \frac{\partial v}{\partial x} .
\end{gathered}
$$

The total strains at a arbitrary distance $z$ of the middle surface are written by

$$
\begin{aligned}
& \varepsilon_{x}=\bar{\varepsilon}_{x}+\kappa_{x} z, \\
& \varepsilon_{s}=\bar{\varepsilon}_{s}+\kappa_{s} z,
\end{aligned}
$$

* Corresponding author: eva.kormanikova@tuke.sk

(C) The Authors, published by EDP Sciences. This is an open access article distributed under the terms of the Creative Commons Attribution License 4.0 (http://creativecommons.org/licenses/by/4.0/). 


$$
\varepsilon_{x s}=\bar{\varepsilon}_{x s}+\kappa_{x s} z
$$

Each individual layer is assumed to be in a state of generalized plane stress, the Hooke's law yields

$$
{ }^{n} \sigma_{i}={ }^{n} E_{i j} \varepsilon_{j}, \quad i, j=(x, s, x s),
$$

where $n$ is number of one layer and $E_{i j}$ is component of elasticity matrix defined in [7].

The force and moment resultants (Fig. 3) are defined by

$$
N_{i}=\int_{-h / 2}^{h / 2} \sigma_{i} d z, \quad M_{i}=\int_{-h / 2}^{h / 2} \sigma_{i} z d z, \quad i, j=(x, s, x s) .
$$

The constitutive equations are written in the matrix form

$$
\left(\begin{array}{l}
\boldsymbol{N} \\
\boldsymbol{M}
\end{array}\right)=\left(\begin{array}{ll}
\boldsymbol{A} & \boldsymbol{B} \\
\boldsymbol{B} & \boldsymbol{D}
\end{array}\right)\left(\begin{array}{l}
\bar{\varepsilon} \\
\boldsymbol{\kappa}
\end{array}\right),
$$

where $\boldsymbol{A}$ is the extension matrix, $\boldsymbol{B}$ is the bendingextension coupling matrix, $\boldsymbol{D}$ is the bending matrix.

The components of $\boldsymbol{A}, \boldsymbol{B}, \boldsymbol{D}$ matrix are

$$
\begin{gathered}
A_{i j}=\sum_{n=1}^{N}{ }^{n} E_{i j}\left({ }^{n} Z^{-}{ }^{n-1} Z\right), \quad B_{i j}=\frac{1}{2} \sum_{n=1}^{N}{ }^{n} E_{i j}\left({ }^{n} z^{2}-{ }^{n-1} z^{2}\right), \\
D_{i j}=\frac{1}{3} \sum_{n=1}^{N}{ }^{n} E_{i j}\left({ }^{n} z^{3}-{ }^{n-1} z^{3}\right)
\end{gathered}
$$

where $N$ is number of layers.
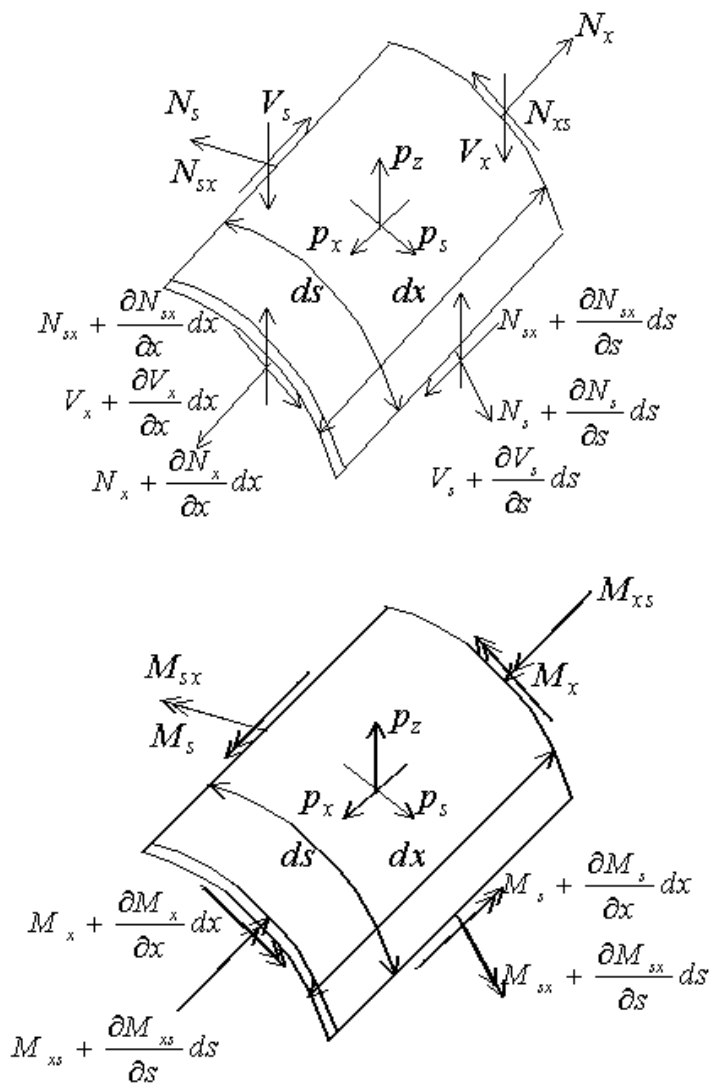

Fig. 3. Force and moment resultants of the differential shell element.
The equilibrium equations for differential shell element on Fig. 3 are given by

$$
\begin{gathered}
\frac{\partial N_{x}}{\partial x}+\frac{\partial N_{x s}}{\partial s}=-p_{x} \\
\frac{\partial N_{x s}}{\partial x}+\frac{\partial N_{s}}{\partial s}+\frac{1}{R}\left(\frac{\partial M_{s}}{\partial s}+\frac{\partial M_{x s}}{\partial x}\right)=-p_{s} \\
\frac{\partial^{2} M_{x}}{\partial x^{2}}+2 \frac{\partial^{2} M_{x s}}{\partial x \partial s}+\frac{\partial^{2} M_{s}}{\partial s^{2}}-\frac{N_{s}}{R}=-p_{z}
\end{gathered}
$$

Substituting the constitutive equations into the equilibrium equations yields a set of three coupled partial differential equations for the three displacements $u, v, w$ which can be written in matrix form

$$
\left(\begin{array}{lll}
L_{11} & L_{12} & L_{13} \\
L_{21} & L_{22} & L_{23} \\
L_{31} & L_{32} & L_{33}
\end{array}\right)\left(\begin{array}{l}
u \\
v \\
w
\end{array}\right)=-\left(\begin{array}{l}
p_{x} \\
p_{s} \\
p_{z}
\end{array}\right) .
$$

The linear differential operators $L_{i j}$ are defined in [5]. The governing equations are solved by the help of Finite Element Method (FEM).

\section{Finite element analysis}

The basic idea of the FEM is a discretisation of the continuous structure. The discretisation is defined by finite element mesh make up of elements nodes. The starting point for elastostatic problems is the total potential energy. In accordance with the Ritz method the approximation is used for displacement field vector by notation

$$
\widetilde{\boldsymbol{u}}(x)=N(\boldsymbol{x}) \boldsymbol{v},
$$

where $N(x)$ is the matrix of the shape functions, that are functions of the position vector $\boldsymbol{x}=(x, s, z)$ and $\boldsymbol{v}$ is the element displacement vector.

For the stresses and strains we obtain from Eq. (9) the Eq. (10)

$$
\begin{gathered}
\sigma(x)=E \varepsilon(x)=E D N(x) v \\
\varepsilon(x)=D u(x)=D N(x) v=B(x) v
\end{gathered}
$$

With the approximation (Eq. 9) the total potential energy is a function of all the nodal displacement components arranged in the element displacement vector $\boldsymbol{v}$. The variation of the total potential energy

$$
\delta \Pi=\delta \boldsymbol{v}^{T}\left(\int_{V} \boldsymbol{B}^{T} \boldsymbol{E} \boldsymbol{B} \boldsymbol{v} d V-\int_{V} \boldsymbol{N}^{T} \boldsymbol{p} d V-\int_{O_{q}} \boldsymbol{N}^{T} \boldsymbol{q} d O\right)
$$

leads to

$$
\delta \boldsymbol{v}^{T}\left(\boldsymbol{K} \boldsymbol{v}-\boldsymbol{f}_{p}-\boldsymbol{f}_{q}\right)=0
$$

where $p, q$ are volume and surface loadings, respectively and $\boldsymbol{K}$ is the symmetric stiffness matrix given by 


$$
\boldsymbol{K}=\int_{V} \boldsymbol{B}^{T} \boldsymbol{E} \boldsymbol{B} d V
$$

The vectors of the volume forces and the surface forces are written by

$$
\begin{aligned}
& \boldsymbol{f}_{p}=\int_{V} \boldsymbol{N}^{T} \boldsymbol{p} d V, \\
& \boldsymbol{f}_{q}=\int_{O_{q}} \boldsymbol{N}^{T} \boldsymbol{q} d O .
\end{aligned}
$$

If the components of $\delta \boldsymbol{v}$ are independent of each other, we obtain from Eq. (12) the system of linear equations

$$
\begin{gathered}
\boldsymbol{K} \boldsymbol{v}=\boldsymbol{f}, \\
\boldsymbol{f}=\boldsymbol{f}_{p}+\boldsymbol{f}_{q} .
\end{gathered}
$$

All equations considered above are valid for a single finite element and they should have an additional index $E$. We have the inner element energy

$$
U_{E}=\frac{1}{2} \boldsymbol{v}_{E}^{T} \int_{V_{E}} \boldsymbol{B}^{T} \boldsymbol{E} \boldsymbol{B} d V \boldsymbol{v}_{E}=\frac{1}{2} \boldsymbol{v}_{E}^{T} \boldsymbol{K}_{E} \boldsymbol{v}_{E},
$$

with the element stiffness matrix

$$
\begin{gathered}
\boldsymbol{K}_{E}=\int_{V_{E}} \boldsymbol{B}^{T} \boldsymbol{E} \boldsymbol{B} d V, \\
\boldsymbol{E}=\sum_{n=I}^{N}{ }^{n} \boldsymbol{E}, \\
{ }^{n} \boldsymbol{E}=\overline{\boldsymbol{T}}^{T}\left({ }^{n} \beta\right)^{(n)} \boldsymbol{E}_{L} \overline{\boldsymbol{T}}\left({ }^{n} \beta\right),
\end{gathered}
$$

where $\boldsymbol{E}$ is the elasticity matrix obtained with suitable transformations in two stages, firstly from the principal material directions to the element local directions and secondly to the global directions. $\boldsymbol{B}$ is the strain matrix, $\boldsymbol{T}$ is the transformation matrix with

$$
\overline{\boldsymbol{T}}(\beta)=\left(\boldsymbol{T}^{T}(\beta)\right)^{-1} .
$$

Because the energy is a scalar quantity, the potential energy of the whole structure can be obtained by summing the energies of the single elements. By a Boolean matrix $\boldsymbol{L}_{E}$ the correct position of each single element is determined. The element displacement vector $\boldsymbol{v}_{E}$ is positioned into the system displacement vector by the equation

$$
\boldsymbol{v}_{E}=\boldsymbol{L}_{E} \boldsymbol{v},
$$

then we obtain the system equation by summing over all elements [8-11]

$$
\left(\sum_{i} \boldsymbol{L}_{i E}^{T} \boldsymbol{K}_{i E} \boldsymbol{L}_{i E}\right) \boldsymbol{v}=\left[\sum_{i} \boldsymbol{L}_{i E}\left(\boldsymbol{f}_{i E p}+\boldsymbol{f}_{i E q}\right)\right] .
$$

The system stiffness matrix is also symmetric, but it is a singular matrix. After consideration of the boundary conditions of the whole system, $\boldsymbol{K}$ becomes a positive definite matrix and the system equations can be solved.

\section{Sizing optimization problem}

The basic problem is the minimization of a function subject to inequality constraints [12]:

Minimize objective function

$$
Z=F(X) \rightarrow \min ,
$$

Subject to constraints

$$
\begin{array}{cc}
\bar{X}_{i}^{L} \leq X_{i} \leq \bar{X}_{i}^{U} & i=1,2, \ldots, N_{d}, \\
g_{j}(X) \leq 0 & j=1,2, \ldots, N_{c},
\end{array}
$$

where $X_{i}$ is a design variable [13].

We make use of the existing response at a number of points in the design space to construct a polynomial approximation to the response at other points. The optimization process is applied to the approximate problem represented by the polynomial approximation

$$
F=a_{0}+\sum_{i=1}^{N_{d}} a_{i} X_{i}+\sum_{i=1}^{N_{d}} b_{i} X_{i}^{2}+\sum_{i=1}^{N_{d}-1} \sum_{j=i-1}^{N_{d}} c_{i j} X_{i} X_{j}+\sum_{i=1}^{N_{d}} d_{i} X_{i}^{3}
$$

where $N_{d}$ is the number of design variables, $a_{i}, b_{i}, c_{i j}, d_{i}$ are coefficients to be determined by a least squares regression.

When the objective function and constraints are approximated and their gradients with respect to the design variables are calculated based on chosen approximation, it is possible to solve the approximate optimization problem. Using the Modified Feasible Direction method (MFD) the solving process is iterated until convergence is achieved. To find the search direction, active and violated constraints have to be identified.

Convergence to the optimum is checked by criteria of maximum iterations and criteria changes of objective function. Besides these criteria, the Kuhn-Tucker conditions necessary for optimality must be satisfied by using the Lagrangian multiplier method. The KuhnTucker conditions are sufficient for optimality when the number of active constraints is equal to the number of design variables and if objective function and all of the constraints are convex. Otherwise, sufficient conditions require the second derivatives of the objective function and constraints [14-16]. Convergence or termination checks are performed at the end of each optimization loop. The optimization process continues until either convergence or termination occurs.

\section{Example and results}

There is needed to find the thickness of the shell roof fabricated from laminate $[0 / 45 /-45 / 90]_{S}$ under constant pressure loading $p_{z}$ in the example (Fig. 4). The material properties of each layer were used from homogenization techniques:

$E_{1}=142 \mathrm{GPa}, E_{2}=10.3 \mathrm{GPa}, G_{12}=7.2 \mathrm{GPa}, v=0.27$, $\rho=1.58$ g.cm ${ }^{-3}$.

Each layer of the laminate has the same thickness ${ }^{n} h$. 


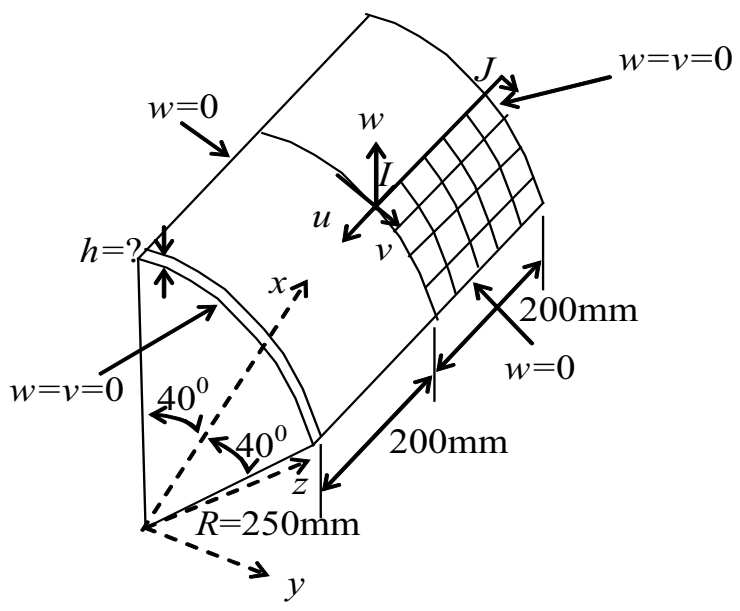

Fig. 4. Problem sketch and finite element model.

Due to symmetry, a quarter of the shell is considered for modelling (Fig. 4).

The mathematical optimization problem we can write: Minimize objective function

$$
F(X)=G\left({ }^{n} h\right) \rightarrow \min [\mathrm{N}]
$$

Subject to constraints

$$
\begin{aligned}
& 0.0125 \leq^{n} h \leq 0.625 \quad[\mathrm{~mm}] \\
& -3.0 \leq w\left({ }^{n} h\right) \leq 0.0 \quad[\mathrm{~mm}]
\end{aligned}
$$

where ${ }^{n} h$ is the thickness of one layer.

The general optimization contains:

1. Initial analysis with input data (Tab. 1).

2. Mathematical optimization problem.

3. Linear approximation.

4. Algorithm of MFD method with convergence criteria.

5. Convergence or termination checks of general optimization.

Table 1. Optimization parameters.

\begin{tabular}{|l|c|c|c|}
\hline $\begin{array}{l}\text { Optimization } \\
\text { parameters }\end{array}$ & $\begin{array}{l}\text { Initial } \\
\text { values }\end{array}$ & $\begin{array}{l}\text { Final } \\
\text { values }\end{array}$ & Tolerance \\
\hline $\begin{array}{l}\text { Design variables } \\
\text { - thickness of one layer } \\
{[\mathrm{mm}]}\end{array}$ & 0.25 & 0.1619 & $1 \cdot 10^{-3}$ \\
\hline $\begin{array}{l}\text { Objective function } \\
\text { - weight [N] }\end{array}$ & 4.4064 & 2.8532 & 0.01 \\
\hline $\begin{array}{l}\text { Constraint } \\
\text { - displacement } w \\
\text { [mm] }\end{array}$ & -1.2478 & -3.0296 & 0.03 \\
\hline
\end{tabular}

Results of the optimization process are listed in the Table 1.

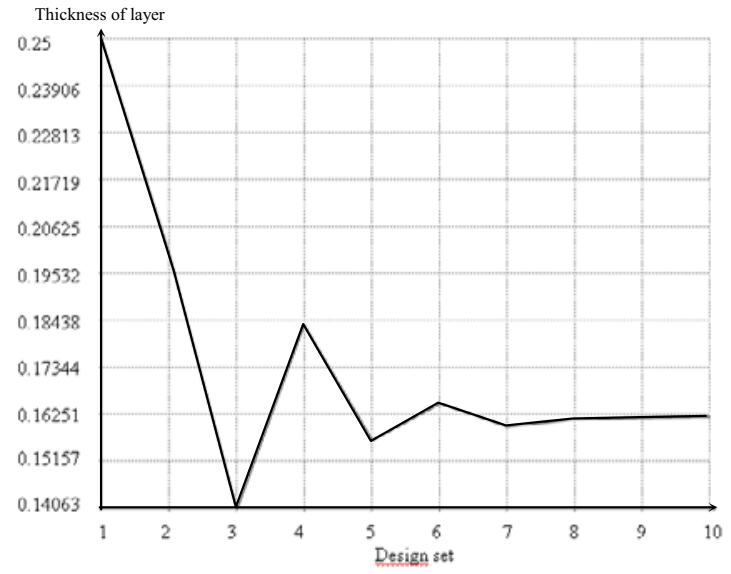

Fig. 5 Variation of the design variable values - thickness [mm] during the optimization process.

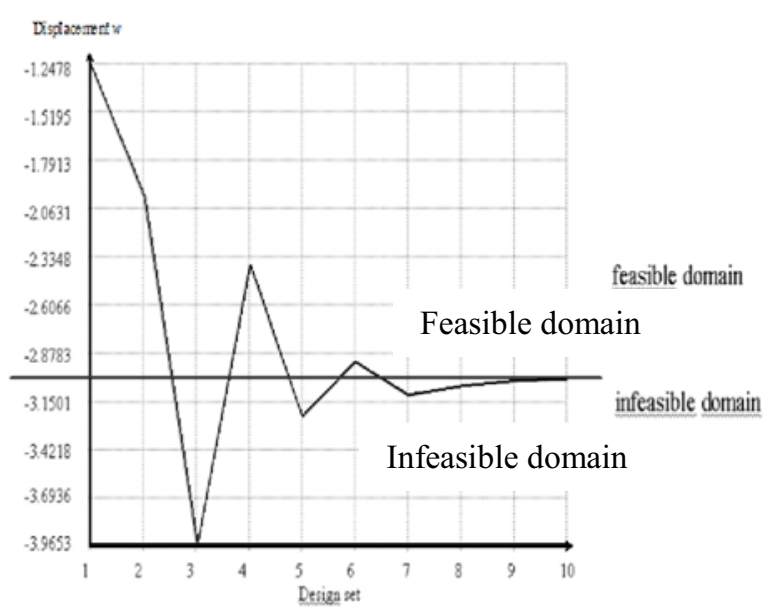

Fig. 6 Variation of the constraint values - displacement $w$ $[\mathrm{mm}]$ during the optimization process.

\section{Conclusion}

The proceeding deals with a numerical approach of modelling of circular cylindrical shell fabricated from fibre composite material. The theory of circular cylindrical shells is described in the frame of classical shell theory. In the paper there are involved the strain displacement relations, constitutive equations and differential equilibrium equations.

There was used homogenization for calculating the material characteristics given in the example [17-19].

In the frame of numerical optimization it was made the minimization of weight subject to displacement constraint. Design variable is thickness of layers of laminate circular cylindrical shell. The initial and final values of design variables, constraints and the objective function are shown in the Table 1.

Maximum number of iterations of MFD was 100. The general optimization process was stopped after 10 design sets (Fig. 5, Fig. 6), because the difference between the current value and the one or two previous designs was less than tolerance specified in the Table 1.

The final value of design variable is ${ }^{n} h=0.16187 \mathrm{~mm}$

(Fig. 5). The total thickness of laminate circular 
cylindrical shell is $8^{n} h=1.3 \mathrm{~mm}$. Figure 6 shows the feasible and infeasible domain in the usable design space.

The optimization is very useful way for design of laminate structural elements including circular cylindrical shells.

This work was supported by the Scientific Grant Agency of the Ministry of Education of Slovak Republic and the Slovak Academy of Sciences under Projects VEGA 1/0477/15 and 1/0078/16.

\section{References}

1. JH. Park, JH. Hwang, CS. Lee, W. Hwang, Stacking sequence design of composite laminated for maximum strength using genetic algorithms, Compos Struct 52, p. 217-31, (2001)

2. S. Adali, V. Verijenko, Optimum stacking sequence design of symmetric hybrid laminates undergoing free vibration. Compos Struct 54, p. 131-8, (2001)

3. G. Soremekun, Z. Gurdal, C. Kassapoglou, D. Toni, Stacking sequence blending of multiple composite laminates using genetic algorithms, Compos Struct, 56, p. 53-62, (2002)

4. PM. Weaver, Design of laminated composite cylindrical shells under axial compression. Composites Part B 31, p. 669-79, (2000)

5. http://file.scirp.org/Html/1-4900125_27735.htm

6. https://www.researchgate.net/publication/26121884 8

7. H. Altenbach, J. Altenbach, W. Kissing, Structural analysis of laminate and sandwich beams and plates. Lubelskie Towarzystwo Naukowe, Lublin, (2001)

8. J. Kralik, Optimal design of npp containment protection against fuel container drop, $A d v$. Mat. Res. 688, p. 213-221, (2013)

9. M. Krejsa, P. Janas, I. Yilmaz, M. Marschalko, T. Bouchal, The use of the direct optimized probabilistic calculation method in design of bolt reinforcement for underground and mining workings, The Scientific World Journal, Article number 267593, (2013)

10. M. Zmindak, Z. Pelagic, M. Bvoc, Analysis of high velocity impact on composite structures, Appl. Mech. and Mat. 617, p. 104-109, (2014)

11. J. Melcer, G. Lajcakova, Comparison of finite element and classical computing models of reinforcement pavement, Adv. Mat. Res. 969, p. 85$88,(2014)$

12. E. Kormanikova, I. Mamuzic, Optimization of laminates subjected to failure criterion, Metal. 50 (1), p. 41-44, (2011)

13. Z. Gürdal, R. T. Haftka, P. Hajela, Design and Optimization of Laminated Composite Materia, J. Wiley \& Sons, (1999)

14. A. M. Valuev, Models and methods of multiobjective optimization in problems of quarry design and planning, WSEAS Trans. on Math., 13, p. 557-566, (2014)
15. E.A. Vorontsova, A projective separating plane method with additional clipping for non-smooth optimization, WSEAS Trans. on Math., 13, p. 115121, (2014)

16. J. Lovíšek, J. Králik, Optimal Control for ElastoOrthotropic Plate, Contr. and Cyber. 2, p. 219-278, (2006)

17. M. Sejnoha, J. Zeman, Micromechanical modeling of imperfect textile composites, Int. Jour. of Eng. Scien. 46 (6), p. 513-526, (2008)

18. J. Ma, S. Sahraee, P. Wriggers, , L. De Lorenzis, Stochastic multiscale homogenization analysis of heterogeneous materials under finite deformations with full uncertainty in the microstructure, Comp. Mech. 55, Issue 5, p. 819-835, (2015)

19. C. Maruccio, L. De Lorenzis, L. Persano, D. Pisignano, Computational homogenization of fibrous piezoelectric materials, Comp. Mech. 55, Issue 5, p. 983-998, (2015) 